\title{
Norois
}

Environnement, aménagement, société

$199 \mid 2006 / 2$

Canada : immigration, espaces urbanisés, parcs nationaux, loisir patrimonial

\section{Identité urbaine, reconversion industrielle et dynamique territoriale à Montréal : le cas d'Hochelaga-Maisonneuve}

Urban identity and spatial dynamics in a post-industrial city: Montreal and Hochelaga-Maisonneuve

\section{Martine Géronimi}

\section{(2) OpenEdition}

Journals

Édition électronique

URL : http://journals.openedition.org/norois/1911

DOI : 10.4000/norois. 1911

ISBN : 978-2-7535-1547-5

ISSN : 1760-8546

Éditeur

Presses universitaires de Rennes

Édition imprimée

Date de publication : 1 juin 2006

Pagination : 45-60

ISBN : 978-2-7535-0332-8

ISSN : 0029-182X

Référence électronique

Martine Géronimi, «Identité urbaine, reconversion industrielle et dynamique territoriale à Montréal : le cas d'Hochelaga-Maisonneuve », Norois [En ligne], 199 | 2006/2, mis en ligne le 13 décembre 2008, consulté le 01 mai 2019. URL : http://journals.openedition.org/norois/1911 ; DOI : 10.4000/ norois.1911 


\title{
IDENTITÉ URBAINE, RECONVERSION INDUSTRIELLE \\ ET DYNAMIQUE TERRITORIALE À MONTRÉAL : \\ LE CAS D'HOCHELAGA-MAISONNEUVE
}

\author{
Martine GÉronimi \\ Groupe de Recherche sur les Espaces Festifs (GREF) \\ UQAM - Université DU QUÉBEC (MonTrÉAL) \\ Geronimi_m@yahoo.ca
}

\section{RÉSUMÉ}

Les villes américaines comme européennes ont expérimenté à l'ère postindustrielle de profonds remaniements économiques et spatiaux. Dans les années 1970, ces villes ont connu une désindustrialisation transformant les quartiers comme Hochelaga-Maisonneuve à Montréal en des zones de pauvreté abandonnées par les industries. Dans les quinze dernières années plusieurs tentatives de reconversion par le tourisme culturel ou les activités festives ont été mises sur pied dans le quartier. Cet article est l'occasion de présenter le quartier Hochela-Maisonneuve ainsi que les différents essais pour le requalifier. Nous essayons de comprendre les raisons pour lesquelles toutes ses interventions furent vaines malgré la détermination et les réalisations des différents acteurs francophones.

Mots clé : Activités ludiques - Centre-ville - Hochelaga-Maisonneuve - Identité - Montréal - Pauvreté - Post-industrialisation - Tourisme culturel.

\footnotetext{
ABSTRACT

Urban identity and spatial dynamics in a post-industrial city: Montreal and Hochelaga-Maisonneuve

In the postindustrialised era, cities in America or in Europe have experienced profound economic and spatial change. In the 70' cities faced the transformation of neighbourhoods, such as Hochelaga-Maisonneuve in Montreal, into poor districts abandoned by industries. In the past 15 years, cultural tourism and festive activities were two ways for the reconversion of the Hochelaga-Maisonneuve's neighbourhood. In this paper, we present the neighbourhood and the attempts to regenerate the district. We try to understand why these interventions were not successful despite the various actions and the determination of the French speaking actors to revitalize this neighbourhood.
}

KEY WORDS : Cultural Tourism - Festive Activities - Montreal - Hochelaga-Maisonneuve - Postindustrialisation - Urban Core - Poverty - Identity. 
Cœur économique du Québec la région de Montréal concentre la majorité de la population et des emplois. La métropole montréalaise, quant à elle, se plait à jouer la carte de l'hyperfestif en se présentant sur la scène internationale comme la capitale des festivals. Le centre de Montréal ressemble à une ville industrielle compacte avec, d'une part des joyaux architecturaux postmodernes, le quartier des affaires à l'ouest, et, d'autre part des quartiers plus pauvres à l'est au patrimoine ouvrier. La rupture entre l'est et l'ouest est marquée par la rue Saint-Laurent, appelée plus simplement « la Main » (rue principale), frontière physique qui matérialise la fracture culturelle entre un ouest anglophone et un est francophone.

La problématique de la reconversion des quartiers industriels désaffectés situés sur l'île de Montréal à proximité du centre ville est un sujet qui préoccupe depuis une vingtaine d'années la métropole à l'instar des autres villes d'Amérique du Nord et d'Europe (Chaline, 1994 ; Charlier et Malézieux, 1994; Vermeersch, 1997 ; Manzagol et Bryant, 1998; Sénécal et Saint-Laurent, 2000; Fontan et al., 2005). Elle est un terrain fructueux également pour les géographes, sociologues urbanistes et économistes qui analysent les cadres de la reconversion économique en tenant compte de l'innovation socio-territoriale (Tremblay et al., 1998; Mustar et Penan, 2003; Klein et al., 1998).

Dans le cas qui nous intéresse, le quartier Hochelaga-Maisonneuve à l'est ${ }^{1}$ (fig. 1), la reconversion socio-économique est délicate car, malgré l'intérêt des acteurs municipaux et provinciaux, malgré la réelle implication du milieu communautaire et en dépit de l'argent injecté, la plupart des tentatives pour animer le quartier et le transformer en un espace vibrant, ayant perdu ses attributs négatifs de pauvreté et de marginalisation, s'avèrent décevantes voire vaines. Le territoire d'Hochelaga-Maisonneuve, produit de l'action collective et de la dynamique des acteurs, reste encore le deuxième quartier le plus pauvre de Montréal après Parc-Extension, un quartier principal d'installation des nouveaux immigrants (Klein et al., 1998).

Or, Hochelaga-Maisonneuve est à $90 \%$ francophone et parle à l'âme des Québécois. Pour le géographe, l'identité est un facteur indispensable à inclure dans sa réflexion s'il veut comprendre les enjeux des acteurs en présence (Cherubini, 1994). Et c'est un quartier qui fait mal à l'âme des Québécois car c'est un des plus pauvres du Canada et cette pauvreté se transmet de génération en génération. Toutes les opérations de revalorisation de l'identité visuelle du quartier sont en grande partie en lien avec une volonté de redonner une fierté aux résidants de ce quartier défavorisé, où le chômage et la misère sont statistiquement évidents. À Montréal, le social est au centre de la problématique de la reconversion et il semble que le concept d'innovation s'applique aux différentes idées mises en œuvre pour redynamiser les territoires en déclin comme Hochelaga-Maisonneuve (Fontan et al., 2005).

L'objet de cet article est de montrer que les projets et expériences de mise en tourisme puis de mise en spectacle de cet ancien quartier industriel, dans un but de requalification, se sont soldés par des échecs successifs malgré des débuts encourageants. Dans un premier temps, après avoir présenté le cadre socio-spatial du quartier, nous examinerons les temporalités de ce quartier. Tandis que la qualité architecturale surprenante du quartier s'explique par sa genèse, son destin industriel n'a fait que suivre les aléas de l'histoire pour aboutir à une désindustrialisation massive, une détérioration du milieu socio-économique et une dévitalisation des artères commerciales. Dans un deuxième temps, nous montrerons combien l'alliance patrimoine tourisme, première dynamique choisie et élaborée sur l'ambition de revaloriser un patrimoine architectural qui repose sur le passé glorieux des fondateurs francophones, n’a pas su convaincre ni les touristes québécois et internationaux, ni les acteurs municipaux. Nous exposerons enfin la seconde dynamique fondée sur les artistes émergents et l'hyperfestif en constatant combien elle a, elle aussi, tourné court depuis 2005. En conclusion, nous esquisserons un bilan du quartier et nous discuterons sur le

1. Il faut souligner que l'échelle paysagère et identitaire retenue dans cet article est celle du quartier. Toutefois il faut savoir, suite à la politique des fusions municipales en 2002, le quartier H-M s'est vu inclure dans l'arrondissement Mercier Hochelaga-Maisonneuve et que depuis les statistiques sont également conjointes; quant aux plans d'action ils reposent sur l'arrondissement et non plus sur le quartier. 


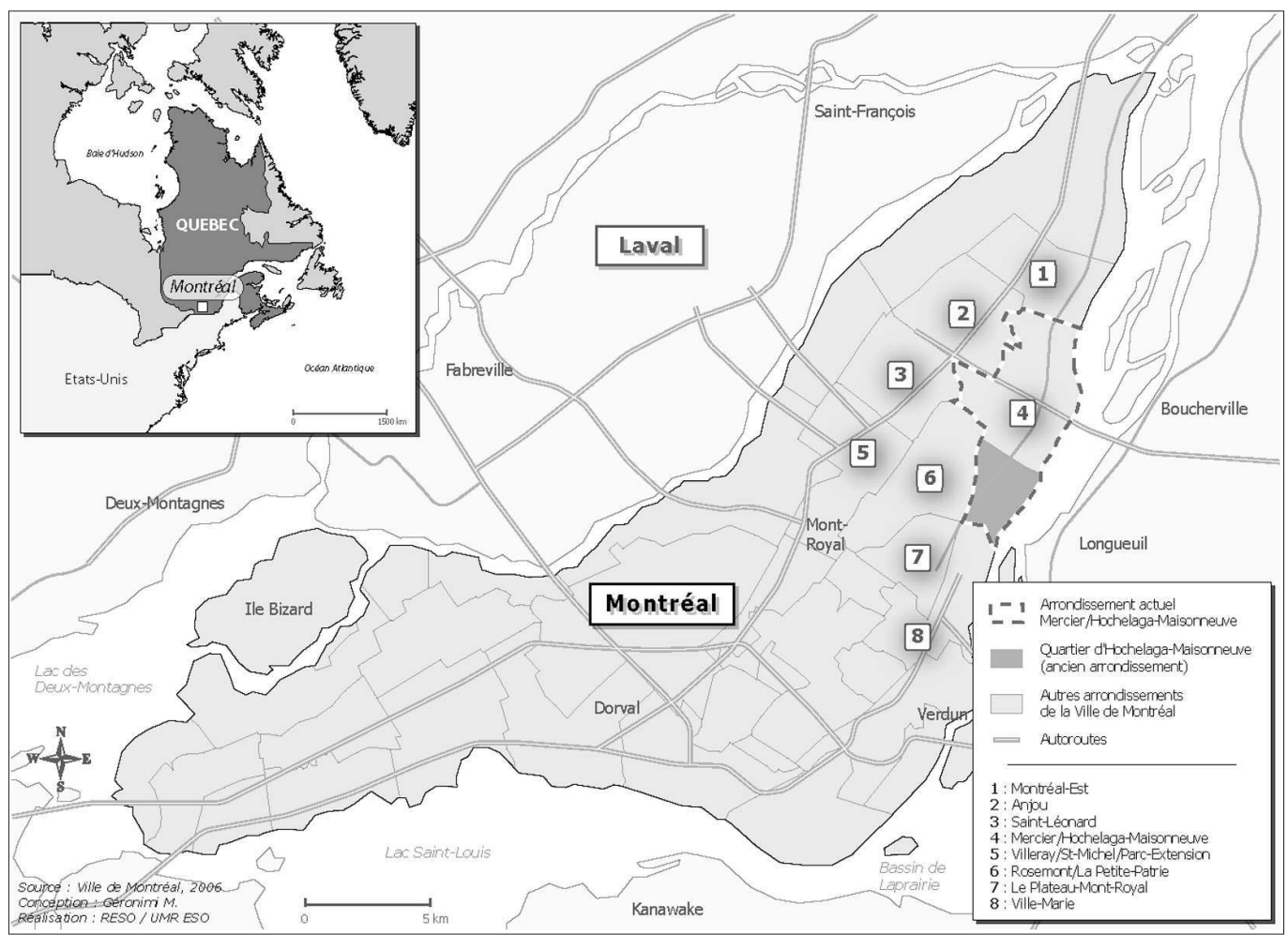

Figure 1 : Localisation du quartier Hochelaga-Maisonneuve dans la Ville de Montréal en 2006 Location of Hochelaga-Maisonneuve district in Montreal city in 2006

concept de patrimoine et les raisons qui sont probablement à la racine de l'échec de l'utilisation du patrimoine comme moteur de la requalification de l'image du quartier. Nous évaluerons ces approches floues et divergentes sous jacentes à la mise en valeur de ce patrimoine.

\section{Hochelaga-Maisonneuve, espace et société}

Nous commencerons en tout premier lieu par une présentation du quartier tel que le découvrent les nouveaux résidants ou les rares touristes s'y promenant. C'est un quartier du siècle dernier au patrimoine architectural intéressant mais vétuste avec des usines désaffectées imbriquées dans le tissu résidentiel. Le paysage social du quartier montre les marques d'une population souvent vieillissante ou jeune et marginalisée, mais surtout relativement pauvre ${ }^{2}$. Ce quartier HochelagaMaisonneuve, inclus depuis la fusion municipale de 2002 dans un plus grand ensemble l'arrondissement MHM (Mercier-Hochelaga-Maisonneuve), couvre un vaste territoire qui conduit du fleuve Saint-Laurent au sud, à la rue Rachel au nord, et de la rue Vimont à l'est, à la rue Moreau à l'ouest. Il compte a priori sur des données favorables : proximité de la dynamique du centre-ville, des grands axes routiers, métro et transport en commun, services publics largement disponibles. De plus, Hochelaga-Maisonneuve bénéficie d’un réseau associatif extrêmement riche en analyses

2. Ce portrait est en train de changer avec 8,2\% d'augmentation de la population dans Hochelaga-Maisonneuve entre 1996 et 2001, surtout redevable à l'arrivée de jeunes travailleurs âgés de 20 à 24 ans, sachant qu'entre 1961 et 1996, il y a eu une diminution de $48 \%$ de la population dans ce quartier (source Plan d'urbanisme de Montréal chapitre de l'arrondissement Mercier-Hochelaga-Maisonneuve). 
et en interventions. Derrière ces données positives, le géographe constate que c'est d'abord un quartier enclavé physiquement : au sud, le port, à l'est la gare de triage et à l'ouest la zone industrielle (fig. 2).

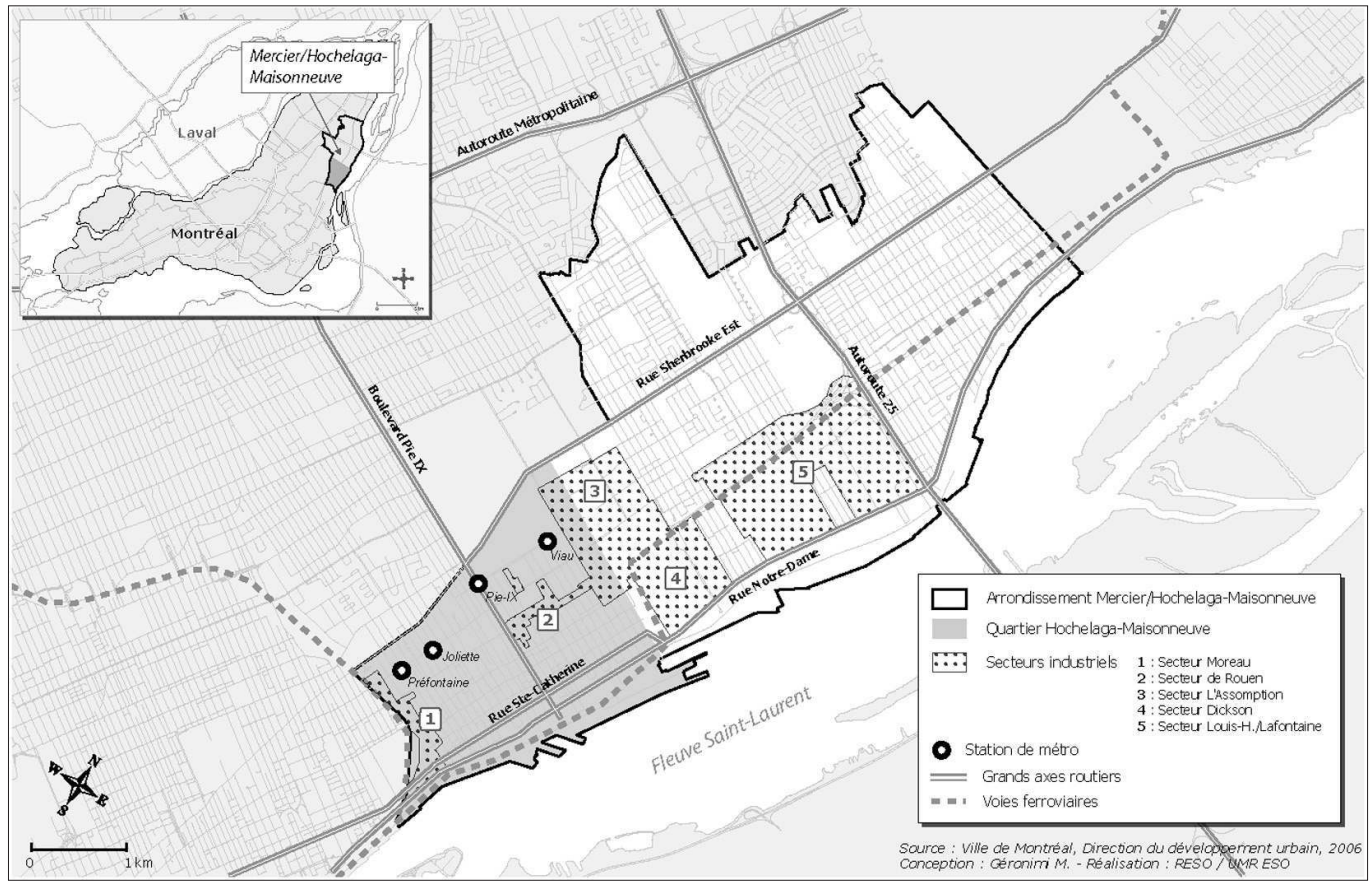

Figure 2 : L'enclavement du quartier Hochelaga-Maisonneuve

Isolation of Hochelaga-Maisonneuve district

Ces problèmes d'enclavement sont liés aux fonctions industrielles qui ont marqué et marquent encore le paysage. Ainsi la zone industrielle (secteur Dickson) à l'est est un vaste secteur de 48 hectares de terrains vacants ou abandonné, plus au nord de Dickson longeant toujours le quartier Hochelaga-Maisonneuve, le secteur industriel de l'Assomption regroupe 29 entreprises et compte 12 ha de terrains vacants (photo 1). De l'autre côté, en plein cœur de Hochelaga-Maisonneuve dans le secteur Rouen entre la rue Viau et la rue Pie-IX, les entreprises manufacturières qui subsistent encore côtoient les secteurs résidentiels et génèrent des nuisances qui entravent le développement de projets résidentiels de gamme supérieure comme le camionnage, bruit, activité industrielle sur le domaine public, etc. (photo 2).

Une forme de gentrification est appelée des voeux des promoteurs immobiliers ainsi que des commerçants, notamment ceux de la rue Ontario où les activités commerciales sont les plus importantes. Mais cette gentrification n'est toutefois pas vue par tous comme la solution pour requalifier le quartier et a même déclenché des actions anti-gentrification à l'hiver 2003-2004 (Myles, 2004). À l'heure actuelle, le secteur a plutôt l'allure d'une friche industrielle composée d'édifices à bureau, d'entreprises de service, d'établissements manufacturiers, d'entreposage et de commerce de gros qui côtoient les milieux résidentiels. La relocalisation de ces entreprises est envisagée dans le plan d'urbanisme de 2004 puisqu'elle « représente aussi une opportunité de dégager des espaces pour accueillir des entreprises qui s'intègrent de façon harmonieuse avec les secteurs résidentiels situés à proximité » (Ville de Montréal, 2004). L'enjeu est de libérer des espaces pour aménager l'accès au Marché Maisonneuve dont la place a été restaurée en 2003. De même, l'autre artère commerçante Sainte-Catherine souffre de défections et ses espaces locatifs 
Photo 1 : Terrains vagues, secteur l'Assomption (cliché : M. Delisle - mai 2006)

\section{District}

Wasteland in l'Assomption

Photo 2 : Rue Notre-Dame, cohabitation entre restaurant et industrie pétrochimique (cliché : M. Delisle - mai 2006)

Notre-Dame street, restaurant in the middle of oil storage tanks
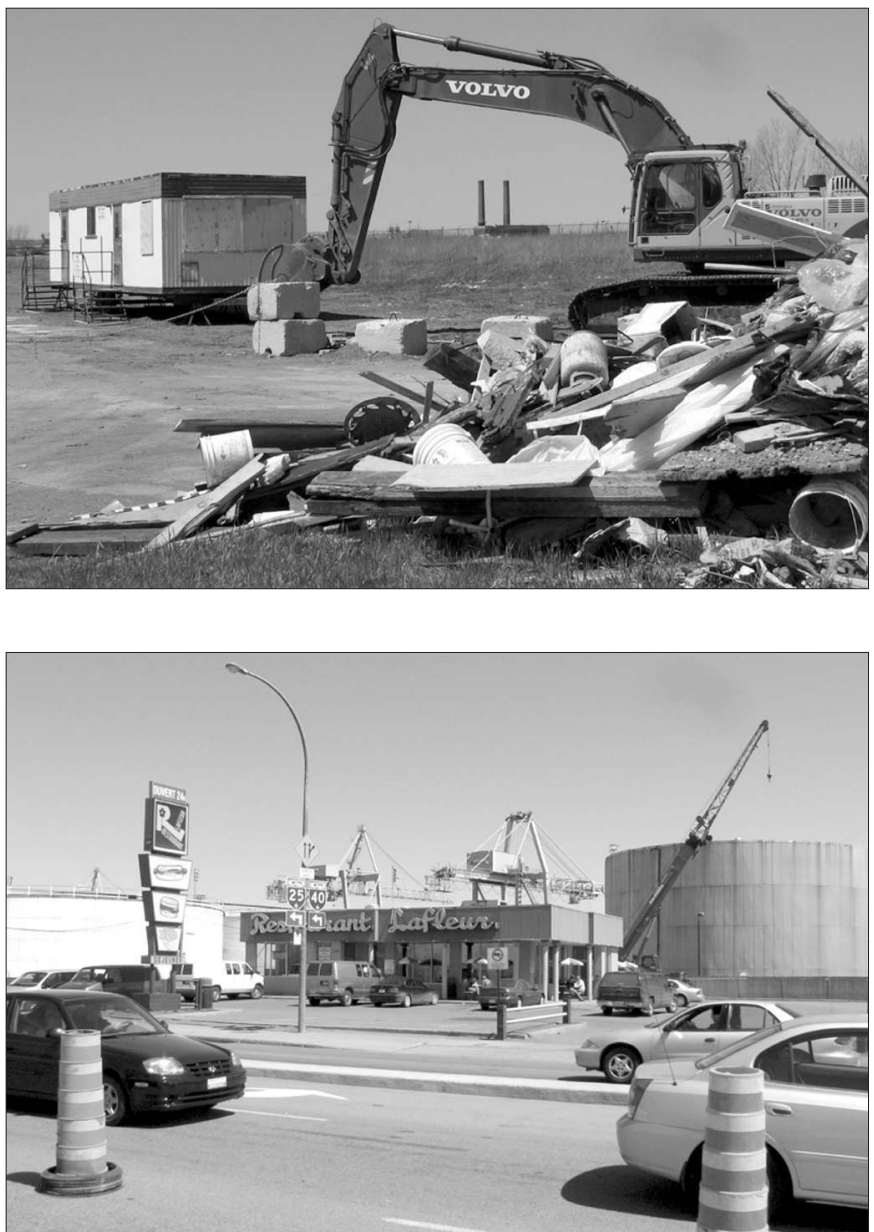

sont vacants à $25 \%$ à l'ouest de la rue Bourbonnière. Ainsi le portrait global du quartier est assez peu attractif avec, selon les secteurs, une déstructuration industrielle complète dans le secteur Dickson, une restructuration en cours dans le secteur de l'Assomption et des problèmes de voisinage et des pressions commerciales et résidentielles.

Le portrait social que l'on peut dresser du quartier passe par plusieurs problématiques tournant autour de la pauvreté. Drogue, prostitution et anciennement gangs de motards criminalisés ${ }^{3}$ sont les fléaux accompagnant une situation d'indigence endémique. Cette dernière se traduit entre autres par : des taux de décrochage scolaire élevés : $43 \%$ des jeunes de 15-24 ans du quartier ne fréquentent plus l'école (la moyenne déjà élevée dans la région de Montréal est de $32 \%$ ), des taux de chômage prolongé plus que préoccupants; ainsi on dénombrait en mars 2004, 29,1\% de la population sur l'aide sociale quand elle est de 12,4\% pour l'ensemble de Montréal, une proportion de 45,9\% des familles vivaient sous le seuil de la pauvreté en 2000 , plus de $51 \%$ étaient composées de famille mono-parentale ${ }^{4}$. À signaler, la proportion des immigrants est passée de $4.8 \%$ à 10,4\%. À ces problèmes de réputation se greffent de réelles questions de santé publique.

3. L'État québécois met un terme aux gangs de motards en janvier 2002 par la loi anti-gang C-24.

4. Statistiques tirées du mémoire du CLSC (Centre local de services communautaires) Hochelaga-Maisonneuve en ligne [http://www.clsc-hochelaga-maisonneuve.qc.ca/memoire_clsc.htm]. 
La population du quartier Hochelaga-Maisonneuve présente un bilan de santé très mauvais : mortalité précoce (72,7 ans d'espérance de vie soit cinq années de moins que la moyenne régionale), taux élevés de maladies respiratoires et de l'appareil circulatoire; taux record régional de suicide (36,6 pour 100000 contre 15,1 pour la région) ${ }^{5}$.

Il existe pourtant des éléments positifs qui viennent tempérer ce tableau alarmant. Héritière d'un passé ouvrier dans un espace villageois, la population a gardé un esprit d'entraide et une bonne capacité à la solidarité. C'est ainsi que le dynamisme communautaire est un facteur de cohésion et de renaissance d'une vie de quartier. Avec plus de 200 organismes communautaires, une dizaine de tables de concertation et grâce à un grand nombre de bénévoles, Hochelaga-Maisonneuve a réussi à se créer une véritable communauté d'appartenance soutenue par des expériences de concertation telles la Corporation de développement de l'est, la CDEST (Corporation de développement de l'est $)^{6}$. Lorsqu'on prend le temps de connaître le lieu et ses habitants, il apparaît que ce quartier ressemble surtout à un village qui serait une entité à part entière dans la Ville de Montréal.

\section{Les temporalités explicatives}

\section{DE LA CRÉATION À LA DESTRUCTURATION}

Le Pittsburgh du Canada, cette ville nouvelle appelée Maisonneuve en l'honneur du fondateur de Montréal a connu un destin hors du commun. Administrée par des esprits enclins à la modernité et aux pensées progressistes, cette cité prospère assez rapidement. La ville est présentée comme un modèle en Amérique du Nord à la fin du XIX siècle; elle devient même en 1912 « les jardins de Montréal ». Ce glissement sémantique suggère alors une double réalité qui se met progressivement en place avec le temps; il s'agit d'attirer les entreprises et la population dans cette banlieue, encore une campagne.

Le premier enjeu fut donc d'installer des services publics dignes d'une ville moderne et attrayante. Dans un souci de marketing, il était nécessaire de rendre cette banlieue la plus séduisante possible. Un plan d'aménagement urbain entraîna la construction d'égouts, la mise en place de réseau de gaz et d'électricité et l'ouverture de routes. De plus, une politique avantageuse de dégrèvement fiscal favorable aux industriels fut votée dès 1885. Les deux premières usines s’installèrent en 1887 et 1888, la St. Lawrence Sugar Refining Co et la Montreal Terra Cotta Lumber, propriété d'Alphonse Desjardins, fondateur des Caisses du même nom, grande banque coopérative fierté du Québec. Elles bénéficiaient toutes deux d'exemption de taxes pour vingt ans. De même, s’installe sur place la savonnerie Barsalou en 1887. En deux ans, la population est multipliée par dix.

En 1896, la construction d'une voie ferrée traversant la ville et raccordée au réseau ferroviaire national stimula la concentration d'industries dans la partie limitrophe au port. Les rues Sainte Catherine et Ontario furent ouvertes et parcourues par les voitures électrifiées du Montreal Street Railway Co, reliant ici Maisonneuve à Montréal. Les nouvelles usines bénéficièrent de conditions très avantageuses avec pour contrepartie l'embauche de résidants de Maisonneuve et une exploitation pendant au moins dix ans de leur entreprise sur place. Ce sont en particulier six usines de chaussures qui s'installèrent. En 1903, la fabrication et la réparation de matériel ferroviaire entraînèrent la construction de l'usine Angus du Canadien Pacifique. Ce sont les années 1910 qui firent mériter le surnom de Pittsburgh du Canada. Sur la commune se centraient alors, les entreprises aussi variées que la Warden King E Son Foundry, les Biscuits Viau, la National Licorice, la Canadian Spool E Cotton, La Canadian Vickers, la United Shoe Machinery, l'American Can, et même Air Liquide Canada etc.

5. Chiffres tirés du site de la Santé publique de Montréal.

6. La ministre Louise Harel, députée de l'arrondissement, soulignait à l'assemblée nationale que 443 bénévoles animaient les 222 organisations communautaires lors de Commission permanente de l'aménagement du territoire du vendredi 19 mai 2000. 
C'est dans cette période que le conseil municipal choisit de se lancer dans une politique de grands travaux et d'embellissement de la ville, inspirée par les principes du City Beautiful Movement. Une équipe composée des deux frères Dufresne et du maire Alexandre Michaud se lancèrent dans cette aventure. Ils constituèrent alors tout le patrimoine d'architecture civile en construisant l'hôtel de ville en 1912, le Marché en 1914, le poste des pompiers en 1915 et en 1916, le bain public et le gymnase (photos 3 et 4). Dès 1909, le Parc de Maisonneuve avait été envisagé suivant en cela les idées hygiéniques et esthétiques nécessaires à la réhabilitation d'un paysage urbain fortement malmené par l'industrialisation galopante de ces années de prospérité économique.

La réalisation de tous ces projets grandioses ne fut pas sans conséquence puisque lors de l'entrée en guerre en 1914, l'économie de Maisonneuve s'en ressentit et la ville fut acculée à la faillite. En 1918, la ville de 37000 habitants se voit annexer à Montréal par décision du gouvernement provincial.

Photo 3 : Bains publics Maisonneuve (cliché : M. Delisle - mai 2006) Morgan Public Bath

Photo 4 : Marché Maisonneuve (cliché : M. Delisle - mai 2006) ket

Original Maisonneuve mar-
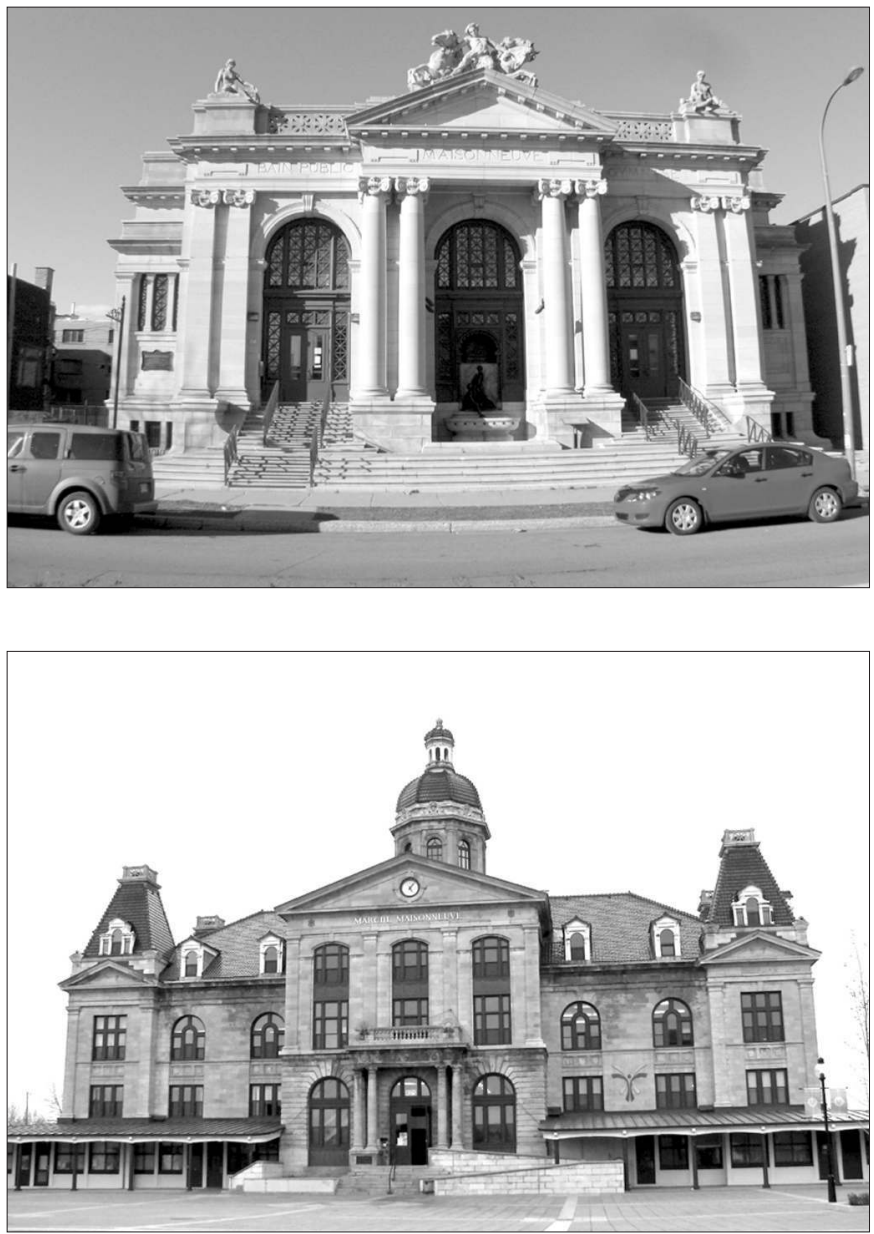

La grande dépression des années 1930 se termine avec la relance économique liée à l'effort de guerre, en particulier grâce aux chantiers navals de La Canadian Vickers. Mais à partir des années 1950, les usines ferment ou se délocalisent, les habitants attirés par les banlieues quittent Hochelaga-Maisonneuve. Le quartier ouvrier, qui lentement se décompose dans les années 1970, 
expérimente la démolition de quelque 2000 logements pour la réalisation du projet d'aménagement de l'autoroute est-ouest.

La déstructuration du quartier est de plus en plus préoccupante et à la fin des années 1980, le tableau est assez dramatique. Il a subi une désindustrialisation majeure à la fois par sa rapidité et son importance. Ce sont les industries du rail et du transport avec des entreprises telles que Vickers puis Angus qui conduisent à l'évidage du quartier car les entreprises soit en partent s'installer vers l'ouest du Canada soit en ferment tout simplement leurs portes comme Angus en 1991 (Fontan et Yaccarini, 1999). De même les industries textiles ou de la chaussure ont quitté le quartier comme la compagnie J.-P. Coats qui employait une abondante main-d'oeuvre féminine vers 1950 et se spécialisait dans la production du fil servant aux machines à coudre. L'usine a fermé définitivement ses portes en 1988. Le quartier se vidant, les industries alimentaires se sont relocalisées ou ont disparu tel le distributeur Steinberg. Toutes ces fermetures ont atteint directement la fierté des résidents du quartier. La nouvelle crise et la restructuration économique des années 1990 entament durement la confiance et l'estime de soi des gens de la communauté du quartier.

\section{LE PATRIMOINE À LA RESCOUSSE DU PRÉSENT; \\ RECONQUÉRIR UNE FIERTÉ, DÉVELOPPER LE SENTIMENT D’APPARTENANCE}

Il est important de voir que le passé du quartier a été valorisé au travers de l'exposition de son bâti architectural. Ainsi. Un des thèmes qui va être pris en compte par les intervenants touristiques au milieu des années 1990 jusqu’à 2003 est justement ce sentiment de fierté et d'appartenance de la communauté pour le territoire qu'elle habite (Lettre UMR ESO, 2005).

Les organisations communautaires ont compris que pour transformer leur environnement, il fallait, outre la bonne volonté des bénévoles, disposer d'outils de développement contrôlés par un leadership local. Ainsi nous pouvons lire dans l'historique du développement des communautés locales que les résidents d'Hochelaga-Maisonneuve «supposent que la solution aux problèmes de la population passe par la constitution d'un leadership local et par la mise en place de services collectifs » (Ville de Montréal, 2000). Le Collectif en aménagement urbain Hochelaga-Maisonneuve, quant à lui, est un organisme communautaire sans but lucratif, qui œuvre dans le domaine de l'aménagement et de l'habitation. Depuis 1996, c'est un des éléments moteurs de la prise en charge du quartier, non pas par l'élite mais par les résidents. Parmi ses champs d'intervention, ce Collectif collabore notamment avec le Service de l'habitation de la ville de Montréal dans le cadre du Programme de revitalisation des quartiers centraux.

Ce développement repose sur la prise en compte de l'aménagement urbain (environnement naturel et bâti) (Gravari-Barbas et Veschambre, 2000). Dans ce dossier, le rôle de la ville de Montréal est important. La reconnaissance de la ville de Montréal de la relation patrimoine tourisme s'exprime en ces termes : «Le patrimoine bâti du quartier Maisonneuve représente incontestablement un atout majeur pour valoriser le quartier et susciter un plus grand intérêt des visiteurs du pôle récréo-touristique »(Ville de Montréal, 1998).

Cette problématique de l'attractivité des lieux est reprise par le gouvernement du Québec qui apporte un vif intérêt à ce quartier défavorisé. Ainsi en 2001, sur le site de Tourisme Québec, un communiqué de presse faisait état de « 2 millions de dollars canadiens pour faire du quartier Hochelaga-Maisonneuve un nouveau pôle touristique d'avant-garde ». En août 2002, c'était au tour du ministre d'État aux Affaires municipales et à la Métropole, à l'Environnement et à l'Eau, Monsieur André Boisclair, d'annoncer que le gouvernement Québécois injecter, en partenariat avec la ville de Montréal, un investissement de quelque 4,71 millions de dollars canadiens pour la première phase d'un grand projet de revitalisation dans Hochelaga-Maisonneuve ${ }^{7}$. Cet afflux d'argent devait permettre, dans l'esprit des édiles gouvernementaux et locaux, de contrebalancer la situation critique dans laquelle se trouvent les habitants du quartier. Concrètement il était ques- 
tion d'aménager une place publique, la place Valois en cours d'achèvement, et de décontaminer de terrains pollués par l'industrie.

\section{Les dynamiques salvatrices}

\section{LA DYNAMIQUE INITIALE, CELLE D'UNE ISSUE À LA CRISE GRÂCE À L’ALLIANCE TOURISME ET PATRIMOINE}

L'originalité incontestée de l'entreprise date de 1995 avec la création d'un organisme communautaire s'attachant à développer la problématique du tourisme culturel dans le quartier : Tourisme Hochelaga-Maisonneuve (THM).

Nous référant au site Internet de l'organisme de l'époque, nous lisions que la mission définie par THM est de "redynamiser le quartier Hochelaga-Maisonneuve, sévèrement touché par la désindustrialisation de l'Est montréalais et la crise économique, en utilisant le levier du développement touristique ». Les membres de THM faisaient référence au tourisme culturel qui « doit favoriser l'instauration d'un climat d'accueil chaleureux, fruit de l'implication de la population dans ce travail de revitalisation ${ }^{8} »$.

Les projets ambitieux de THM s'étaient vus confirmer en 1997 par une étude d'un cabinet conseil spécialisé en développement touristique ${ }^{9}$ qui certifiait de la richesse patrimoniale exceptionnelle du quartier reposant sur une forte concentration de bâtiments de style Beaux-Arts, un patrimoine industriel du début du siècle et un patrimoine religieux intéressants. Cette étude faisait de plus référence à la proximité du pôle Maisonneuve (parc olympique, jardin botanique, insectarium, biodôme) qui attire chaque année plusieurs millions de visiteurs. Fort de ces avis prometteurs, l'organisme qui regroupait une quarantaine de membres corporatifs et individuels, s'est lancé dans une politique d'animation des lieux selon trois axes principaux : la mise en valeur du patrimoine, l'animation culturelle et l'ambiance de quartier. Le but ultime était d'inscrire Hochelaga-Maisonneuve parmi les principaux attraits touristiques montréalais.

Toutefois cette politique d'animation des lieux a connu un échec généralisé après quelques années. Animation culturelle et ambiance de quartier sont les volets qui paraissaient les plus en lien avec la problématique sociale du quartier mais il s'est avéré qu'ils étaient aussi les plus fragiles.

\section{Des ÉCHECS SUCCESSIFS, LA BOLDUC, Maurice Richard et le circuit Guido Nuncheri}

Sur les traces de la bolduc ${ }^{10}$, un circuit animé au travers du quartier, est un spectacle qui a effectivement duré 4 années, pendant lesquelles cinq fois par semaine, dans les rues du quartier Hochelaga-Maisonneuve, une jeune gaspésienne, Marie Travers dite La Bolduc, venait à la rencontre de l'histoire et de son public. Ce spectacle, initialement chanté par deux femmes artistes locales ${ }^{11}$, était une création d'une autre femme, la metteure en scène Lorraine Beaudry ${ }^{12}$. Cette animation de rue était une pièce de théâtre de rue en dix tableaux durant lesquels comédiens et public interagissaient. Ainsi le public pouvait connaître l'œuvre et les lieux de vie de la plus célèbre chanteuse québécoise d'avant-guerre. Cette chanteuse a valeur de symbole pour ce quartier populaire de l'Est de Montréal mais aussi pour l'ensemble des québécois francophones. Elle est la première femme auteure-compositeure-interprète du Québec et du Canada français (Dufour, 2001).

\footnotetext{
8. Site web disparu depuis la fermeture définitive de THM

9. Pensum conseil Inc. dont le président est Paul Arsenault, également Directeur du Réseau de veille en tourisme de l'UQAM

10. Pour l'histoire de la célèbre chanteuse La bolduc, information disponible sur internet : [http://www.qim.com/artistes/biographie.asp?artistid=46] (dernière consultation 18 avril 2006)

11. Angèle Poirier, puis repris par Monique Maisonneuve.

12. Actuellement directrice générale du théâtre Le rideau Vert sur la rue St-Denis dans le quartier du Plateau Mont-Royal.
} 
Ce spectacle en pleine rue réalisait pleinement les objectifs de THM. Il réussissait le tour de force d'allier les trois problématiques originelles, soit une animation culturelle du quartier avec une ambiance de re-création de la vie passée, celle de l'entre-deux guerres, et une visite patrimoniale des lieux en commençant par la place du Marché. Malgré ces atouts, le circuit piétonnier fut abandonné en 2002, faute de rentabilité. L'initiative de le remplacer par un spectacle, le Cabaret de la Bolduc qui devait être proposé à la clientèle touristique, entre les mois de mai et septembre, a également fait faillite. C'est dans une salle du théâtre Zest, relocalisé dans une ancienne caserne de pompiers entièrement rénovée sur la rue Ontario, que devait se donner un vendredi par mois ce spectacle. Mais la salle de spectacle Zest a elle-même disparu dans la tourmente de toutes les faillites culturelles du quartier et notamment celle des Espaces émergents dont nous parlerons dans la dynamique du patrimoine immatériel.

L'autre personnage québécois remplissant de fierté les résidants d'Hochelaga-Maisonneuve est le célèbre joueur de Hockey Maurice Rocket Richard ${ }^{13}$. Le site de THM faisait référence à L’Univers Maurice Rocket Richard, une exposition thématique permanente sur la vie et la carrière du Rocket Maurice Richard. Cette idole d'au moins cinq générations d'amateurs de hockey au Québec s'est éteinte en 2000. La salle d'exposition, située dans l'Aréna Maurice-Richard qui présentait des objets, des extraits visuels et sonores, des photographies et des coupures de journaux a été fermée à la suite de son décès. Les effets de Maurice Richard ont été rachetés par Patrimoine Canada et la légende vivante s'est vue réapproprier par le Musée Canadien des Civilisations qui, dès avril 2004, a présenté à Ottawa la collection des 75 pièces rachetées à la famille. Cette exposition devrait devenir une exposition itinérante à travers tout le Canada. En attendant, là encore le virtuel rattrape la réalité et nous pouvons retrouver ce grand joueur sur plusieurs sites officiels ou non et en particulier celui de la ville de Montréal ${ }^{14}$. Il apparaît clairement que les résidents d'Hochelaga-Maisonneuve ont perdu une fois de plus dans cette bataille.

De même, un autre circuit culturel et patrimonial a été abandonné durant l'été 2002, celui de «Guido Nincheri, l'alchimiste du vitrail » (Micillo Villata, 1995). Ce maître italien est connu pour ses fresques et vitraux réalisés à Montréal et dans sa région. Montréal abrite 29 églises ou chapelles décorées par Nincheri mais ces réalisations sont considérables à travers tout le Québec et le Canada. Or, le quartier Hochelaga-Maisonneuve possède plusieurs de ses œuvres, notamment à l'église SaintClément-de-Viauville et au Château Dufresne. Ce circuit présentait à la fois le patrimoine civil et religieux du quartier. En effet, cet artiste de renommée international a décoré la demeure des richissimes industriels de la chaussure, les Frères Dufresne, et la seconde paroisse de Maisonneuve dans le quartier réalisé par l'industriel, Charles-Théodore Viau. Mais depuis l'abandon de ce circuit, la meilleure manière de voir les réalisations de cet artiste reste la navigation sur Internet ${ }^{15}$.

Le patrimoine religieux quant à lui est important dans Hochelaga-Maisonneuve, toutefois la difficulté réside surtout dans le recrutement de guides compétents. Ainsi la visite patrimoniale est toujours délicate car la bonne volonté et le bénévolat ne suffisent pas. L'Atelier d'histoire Hochelaga-Maisonneuve, à certains moments, réalisait des visites des églises patrimoniales du quartier en insistant sur les merveilles que sont les vitraux de Nincheri, mais aussi les sculptures de Carli et Petrucci, l'architecture de Joseph Venne et le très bel orgue Casavant de l'Église du Très-Saint-Nomde Jésus de la rue Adam. Une telle initiative n’a jamais été transformée en activité régulière.

\section{UN NOUVEL ESPOIR DÉÇU :}

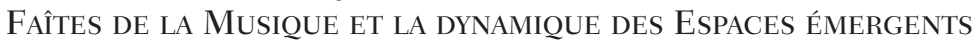

Il nous faut introduire un autre acteur qui avait pris en 2003 le contrôle de l'animation du quartier et qui pendant longtemps était un joueur associé à THM. Il s'agissait de FDM et des Espaces

13. Surnommé le héros des Francophones voir [http://www.angelfire.com/celeb/Rocket/article2.html] (dernière consultation le 17 avril 2006).

14. Celui de la mairie de Montréal, consulté le 17 avril 2006 [http://ville.montreal.qc.ca/portal/page?_dad=portalछ_ pageid=165,230392E_schema=PORTAL].

15. [http://collections.ic.gc.ca/nincheri/fr-main.html] dernière consultation le 17 avril 2006. 
Émergents. Le quasi-abandon du site Internet de THM en 2003 laissait supposer des problèmes au sein de l'organisme. À la fin du mois de mars 2003, la fermeture totale de THM était confirmée. En effet, une crise financière s'est transformée en banqueroute lorsqu'une querelle entre la mairie centrale et THM s'est accentuée en décembre 2002 par la remise en question du financement des concerts populaires. Le sort de THM était scellé. L'affaire de la Joute, sculpture fontaine de l'artiste Riopelle, a fait couler beaucoup d'encre en novembre $2002^{16}$ et a approfondi le gouffre, laissant la place à l'équipe concurrente de FDM-Espaces émergents.

Ainsi, s'envolait la plupart des réalisations initiées par THM. Le Bureau d'accueil touristique ne recevrait plus ses milliers de visiteurs estivaux sur le site de la Place du Marché Maisonneuve. Quant au guide touristique, qui faisait rayonner les attraits patrimoniaux du quartier, depuis plusieurs années, il a été également abandonné. La présence de l'équipe de Tourisme Hochelaga-Maisonneuve lors des salons touristiques, dans le but de promouvoir le quartier comme lieu d'accueil pour visiteurs et touristes, n'est évidemment plus de mise après le licenciement des 7 employés.

Cette mise en valeur difficile du quartier reposait en 2003 sur le dernier volet, celui de l'ambiance de quartier et l'animation de l'espace festif et artistique. Les pouvoirs publics, en l'occurrence la ville centre de Montréal, semblaient avoir tranché en accordant toute son attention et ses aides à la relève des artistes et en fermant THM. En fait, le quartier Hochelaga-Maisonneuve n'avait pas besoin de nouvel échec, «la députée péquiste du quartier souhaitait qu'une nouvelle table de concertation sur le tourisme soit mise sur pied afin de reprendre certaines des activités de promotion et de production d'événements qu'assumait THM ».

À l'été 2003, les festivals s'étaient enchaînés les uns après les autres avec La Grande Rencontre, les Fantaisies et Rythmes Latino américain, le Festival International Country-Western de Montréal, le Festival des Musiques, des Danses, des Échasses et des Masques et des Ballets nationaux et la Fête des enfants de Montréal. À l'automne, quatre manifestations festives avaient tenu le haut de l'affiche : Les Journées de la culture, le Festival Orgue et couleurs, le Carnaval'Est de l'Halloween et le Coup de coeur francophone. Au fondement de cette programmation hyperfestive un choix politique qui favorise une entreprise d'économie sociale pour gérer celle-ci. En effet, l'ensemble des festivals se retrouvait sous l'égide de FDM - Espaces-émergents, " une entreprise d'économie sociale qui a comme mission de participer au développement de la démocratie culturelle au service de la communauté et des artistes des cultures émergentes ${ }^{17}{ }$. Comme le soulignait la ministre péquiste Louise Harel en 2001 : «La nouvelle donne culturelle lancée par Espaces émergents est de créer un lien entre la culture et le social par l'interdisciplinarité et la démocratisation de la culture afin de reconnaître la culture dite marginale, la culture émergente. »

Ces projets devaient être soutenus par le gouvernement mais les changements de majorité en avril 2003 n'ont pas profité à ces initiatives dans un quartier difficile qu'on tentait de requalifier ${ }^{18}$. Le groupe FDM-Espaces émergents qui semblait sortir gagnant de ce conflit en 2003 n'a pas non plus su résister à la déliquescence du quartier. Ce choix louable mais abstrait de favoriser les artistes et la culture émergente avait été favorisé au détriment de la mise en tourisme du patrimoine architectural, préalablement soutenue. Ce changement vigoureux de cap a fait suite à un plan de communication digne des «Success stories » d'entreprises capitalistes ${ }^{19}$. Le résultat du choix de l'hyperfestif et du soutien aux artistes s'expliquait par les 55 groupes culturels actifs et un budget de fonctionnement total de près de $20 \mathrm{M}$ \$. Les trois principaux groupes se situaient à la Caserne Ontario, pour FDM-Espaces émergents, à la caserne Létourneux, bientôt occupée par le Théâtre

16. Dossier Culture Montréal 2002 consultation sur le site de l'association [http://www.culturemontreal.ca/positions/021109op_ lajoute.htm].

17. FDM-Espaces Emergents (2002) ACTES du Forum Actions culturelles et sociales : Espaces émergents Montréal 6 et 7 octobre 2001

18. En juillet 2003, le gouvernement libéral refusait d'honorer la subvention de 700000 dollars promise par le gouvernement Landry pour financer Les Espaces émergents.

19. Notation fiduciaire - Faites de la musique! obtient la note de passage, Bernard Lamarche, Le Devoir, Édition du samedi 7 et du dimanche 8 décembre 2002. 
Sans Fil et dans l'ancien édifice de l'American Can pour Zone-Est Création. Mais cette réussite à l'américaine s'est dégonflée comme un ballon de baudruche (photo 5). Ainsi on pouvait lire (Plan d'action local pour l'économie et l'emploi, 2005):

«Enfin, on a assisté au cours des deux dernières années à la disparition de quelques entreprises et regroupements "phares" en économie sociale : Faites de la musique, Tourisme HochelagaMaisonneuve, la Joujouthèque, Entrée libre et la Luciole (entreprises d'insertion). En conclusion, plusieurs entreprises et organismes en sont rendus à une étape de développement qui les a conduits à une croisée des chemins. [...] La disparition d'entreprises et d'organismes en culture et en tourisme (par exemple, Faites de la musique, Tourisme Hochelaga-Maisonneuve) est ressentie comme une perte importante pour le quartier et sème le doute chez plusieurs quant à la viabilité de la culture (ou, du moins, du tourisme culturel) et du tourisme comme axes potentiels de développement local. »

Et le quartier d'abandonner les logiques de mise en tourisme préconisées une dizaine d'années plus tôt par les experts des cabinets conseils.

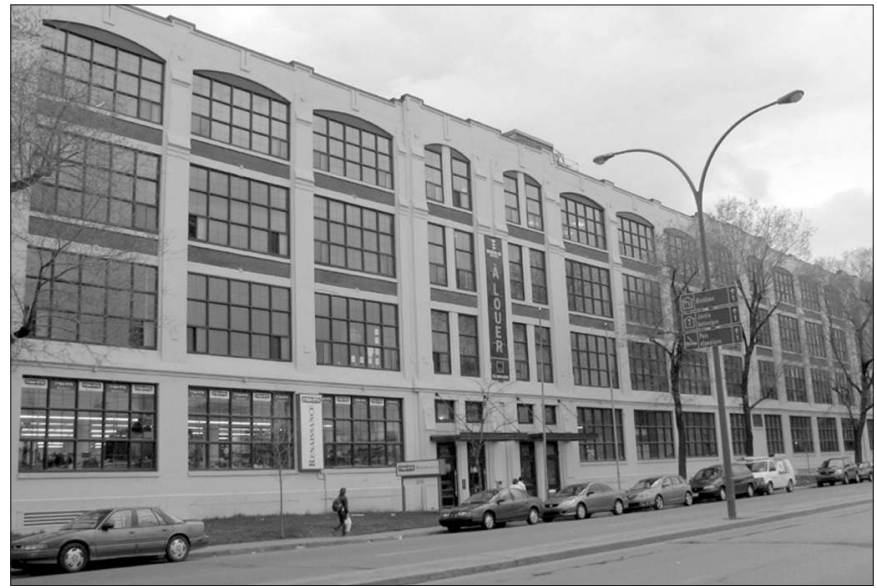

Photo 5 : Lofts dans l'ancienne usine American Can Pie IX (cliché : M. Delisle - mai 2006) tory on Pie-IX boulevard

\section{Conclusion}

\section{L'ÉTAT DU QUARTIER D'UN POINT DE VUE FESTIF : LE FESTIVAL Orgue eT COUleurs, unE LONGÉvité CULTURELlE}

Le Québec s'enorgueillit de très grands facteurs d'orgue comme le célèbre Joseph Casavant. Un orgue Casavant, rénové en 1999 dans l'Église du Très-Saint-Nom-de Jésus, est à la naissance d'un festival qui allie le patrimoine religieux et l'animation culturelle, le festival Orgue et couleurs ${ }^{20}$. De tout le courant d'initiatives culturelles initiées pour requalifier le quartier, seul subsiste encore ce festival, Orgue et couleurs, qui atteint en 2006 sa huitième année. Il faut signaler que cet événement musical dépasse la simple animation du quartier Hochelaga-Maisonneuve. Il semblait toutefois être une fierté de THM par sa réussite, année après année, et la mise en valeur de trois des quatre églises du quartier. En 2002, 35 églises montréalaises ont participé à l'événement Orgues Ouvertes et en 2003, diverses activités satellites ont élargi l'audience dans le temps et l'espace. Le festival automnale attire sur une dizaine de jours 7000 amoureux de l'orgue et se veut désormais une rencontre internationale. 
La question reste de savoir si les résidants d'Hochelaga-Maisonneuve profitent réellement de ces animations pour mélomanes et amateurs de musique baroque et sacrée. On sait combien ce type de musique attire les Anglophones, il serait intéressant d'avoir les chiffres de fréquentation répartie entre les deux communautés, sachant que le quartier est à $90 \%$ francophone. Mais il est certain que la programmation et les prix accessibles voire gratuits des concerts plaident pour l'élargissement du public et l'accessibilité aux personnes du quartier. " La culture est un droit. Elle évite de tomber dans le côté ghetto et permet de s'ouvrir aux autres ${ }^{21}$. Quant aux Concerts populaires de Montréal organisés depuis 40 ans certains mercredi soir et qui avaient été créés à l'initiative du maire Jean Drapeau, ils semblent l'ancrage initial et le ciment de la réussite du festival puisque de tout Montréal convergent les mélomanes et autres néophytes pour écouter les choix musicaux de l'Orchestre symphonique métropolitain. Cette longévité-là repose sur un désir concret de la population de partager la grande Musique.

\section{L'AVENIR DU DÉVELOPPEMENT LOCAL, LA DONNE CULTURELLE}

Le choix de la ville via la Corporation de développement de l'est s'aligne sur le Culturel (CDEST, 2005):

«Plusieurs facteurs militent en faveur du renforcement de la culture comme axe de développement local : la présence d'une masse critique d'artistes, d'entreprises et d'organismes, surtout dans le quartier Hochelaga-Maisonneuve (notamment en arts visuels et en arts d'interprétation); une table de concertation; un potentiel patrimonial; des lieux et des infrastructures de qualité pouvant accueillir des artistes, des regroupements ou des entreprises ».

Le projet structurant autour de ce retour au culturel est l'aménagement de la Maison de la Culture Maisonneuve :

« La ville de Montréal va procéder au cours des prochains mois à la transformation de la Caserne (anciennement Le ZEST), située à l'intersection des rues Ontario et Desjardins, pour y implanter la Maison de la culture Maisonneuve. Cet édifice abritera une salle de diffusion de 300 places, des aires d'exposition et d'animation culturelle ainsi que les bureaux administratifs de la Maison de la culture. Des espaces pourraient être éventuellement offerts à des organismes culturels du quartier. »

Cette maison de la Culture a été inaugurée en septembre 2005.

\section{LA RÉFLEXION SUR LA MISE EN VALEUR DU PATRIMOINE}

\section{A ÉTÉ INSUFFISAMMENT ACCOMPAGNÉE}

À notre avis, le constat d'échecs successifs reliés à la mise en tourisme du quartier reposant sur le culturel est discutable. Il nous faut insister sur le patrimoine de qualité que l'on trouve dans Hochelaga-Maisonneuve. Dans le cas de THM, il nous semble plus juste de parler d'une mauvaise mise en tourisme à un moment où les conditions gagnantes étaient loin d'être réunies. Difficile synchronisation, excès d'optimisme, méconnaissance du milieu touristique, mauvaise gestion... tous ces défauts n'ont pu être surmontés malgré la passion de l'équipe et le bénévolat admirable de bon nombre d'intervenants. Il ne faut pas oublier, d'autre part, qu'au Québec la réflexion institutionnelle sur le patrimoine et sa mise en valeur sont récentes (Drouin, 2005). Ainsi, ce n'est qu’en 2000 que le gouvernement québécois publie « la Déclaration québécoise du patrimoine ». Il faut attendre 2005 pour voir éclore trois bases de réflexion solide sur le patrimoine, l'une universitaire d'envergure internationale, l'Institut du patrimoine à l'Université du Québec à Montréal, la seconde, municipale, avec la Politique du Patrimoine de la ville de Montréal et enfin la troisième

21. Propos relevé dans le journal Le Devoir et tenu par l'un des organisateurs, Pierre Larivière lors d'un entretien en septembre 2002. 
associative (Ville de Montréal, 2005a), le RPM (Réseau patrimoine Montréal), unissant les différents organismes tant privés que publics en partenariat cherchant à optimiser leurs actions de promotion (Ville de Montréal, 2005b).

En réalité localement, la réflexion sur le patrimoine d'Hochelaga-Maisonneuve a été portée par l'Atelier d'Histoire d'Hochelaga-Maisonneuve depuis 1978. Mais maintenant au sein du RPM, cet organisme à but non lucratif aura sans doute plus d'appuis pour réaliser une mise en valeur du patrimoine industriel et urbain du quartier. Il nous semble assez utopique dans un quartier qui se classe parmi les plus pauvres du Canada de mettre l'emphase sur la rentabilité des activités communautaires. En fait, le tourisme patrimonial pourrait renaître dans le quartier lorsque les assauts des promoteurs et les différentes coalitions locales auront réussi à transformer la réputation répulsive du quartier auprès de nouveaux résidents et que le spectre du quartier ne sera plus le mythe ouvrier de la misère mais le nouveau lieu de la bohème branchée montréalaise. En effet, le quartier se gentrifie peu à peu depuis 2004 et les projets se succèdent avec des lofts et de nouveaux condominiums (photo 6). Ce qui nous apparaissait en filigrane en 2003 dans ce dossier se réalise peu à peu : un projet de revitalisation du quartier qui va probablement à long terme entraîner un embourgeoisement des lieux et une récupération de l'espace par les promoteurs immobiliers attirés par les faibles coûts du quartier, un peu à la manière du quartier Saint-Roch à Québec (Simard et Mercier, 2003). Ainsi, une transformation radicale de l'identité du quartier sur les plans physique, social, économique, culturel et démographique va s'ensuivre et le quartier sera complètement transfiguré. D'ici là que feront les artistes?

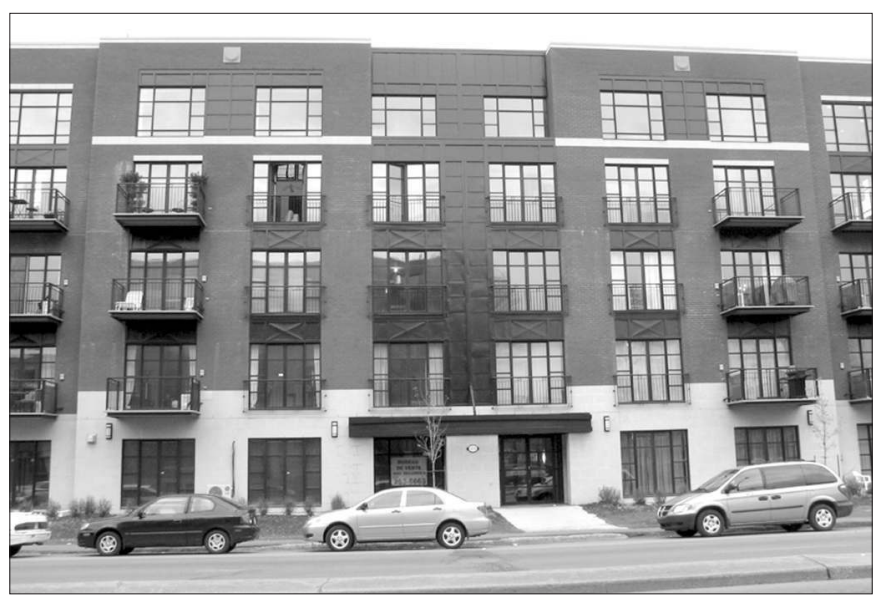

Photo 6: Nouvelle copropriété boulevard Pie IX (cliché : M. Delisle - mai 2006) IX boulevard

New condominiums on Pie-

En attendant, comme le soutient P. Murray dans Après l'histoire (Muray, 1999), reprenant et développant des idées abordées dans les années 1960 par des sociologues français comme J. Dumazedier (Dumazedier, 1962) ou H. Lefebve (Lefebvre, 1968), mais aussi par G. Debord dans "La société de Spectacle », on peut comprendre Hochelaga-Maisonneuve comme l'échec de la recette hyperfestive.

Ainsi, il nous semble qu'au Québec, on peut sans faillir reprendre les mises en garde de Debord lorsqu'il fustigeait «cette époque qui se montre à elle-même son temps comme étant essentiellement le retour précipité de multiples festivités, est également une époque sans fête [...] Quand ses pseudo fêtes vulgarisées, parodies du dialogue et du don, incitent à un surplus de dépense économique, elles ne ramènent que la déception toujours compensée par la promesse dune déception nouvelle» (Debord, 1976). A. Roy, directeur de la revue littéraire L'inconvénient, parle du « tapage festivalier » (Baillargeon, 2005) quand J. Proulx s'insurgeait en 1971 contre une vie quotidienne dans laquelle se dilue la fête, ou du moins des « débris de la fête » (Proulx, 1971). Cette « fièvre 
de l'hyperfestif » montrée du doigt publiquement par le journaliste S. Baillargeon du journal Le Devoir en juin dernier à Montréal, ville qui s'autoproclame capitale mondiale des festivals (Harter, 2005), reprend la critique acérée, menée par des littéraires comme Lakis Proguidis autour de sa revue L'Atelier du Roman qui dénonce cet état de fait si répandu dans nos sociétés post-modernes de ce qu'a nommé P. Muray la « festivisation globalisée » (Muray et Levy, 2005).

La marchandisation de l'espace public n'a cependant pas encore réussi à Hochelaga-Maisonneuve. Il faut toutefois souligner qu'une seule fête est significative sur cet espace, une authentique fête identitaire celle du 24 juin, ancienne fête de la Saint-Jean-Baptiste devenue Fête Nationale du Québec en 1977. Cette dernière a lieu en plein air au parc Maisonneuve et regroupe chaque année des dizaines de milliers de Montréalais francophones dans une messe publique qui célèbre la réussite de la reprise en main de leurs destins par les Francophones du Québec. Une fête qui, elle a un sens profond. En revanche, les essais réitérés par le parti québécois, et notamment la députée de l'arrondissement Louise Harel depuis 1989, de tout faire pour sauver le quartier Hochelaga-Maisonneuve n'ont pas abouti en ce qui concerne la recette hyperfestive. Madame Louise Harel, désormais chef de l'opposition officielle au parti libéral de Jean Charest, continue de défendre son arrondissement mais les crédits, largement prodigués aux différents groupes communautaires, du temps où elle était en contrôle, ne sont plus légion. Si le parti québécois revient au pouvoir, on peut s'attendre à revoir fleurir les subventions, mais pas forcément avec de meilleurs résultats.

Ne doit-on pas s'interroger in fine sur le bien-fondé du festif à tout prix comme moyen de relever 1 »estime des habitants d'un quartier? Ne cherche-t-on pas involontairement à panser des plaies mal cicatrisées avec les mauvais outils? Il nous semble qu'il y aurait réellement matière à une recherche universitaire d'envergure, au-delà de celle de 2000, non pas menée seulement par les réseaux amicaux, ni uniquement en fonction d'intuitions sincères mais réalisée par une équipe transdisciplinaire de chercheurs indépendants préoccupés par l'innovation sociale et la requalification urbaine sans implication partisane dans le milieu. Alors peut-être des solutions véritablement nouvelles émergeront-elles?

\section{Bibliographie}

Baillargeon (S.), 2005. - «La fièvre de l'hyperfestif », Le Devoir, 25-26 juin, p. Al et 10.

CDEST, 2005. - Plan d'action local pour l'économie et l'emploi (PALÉE) 2005-2008, Stratégie de développement économique de l'arrondissement de Mercier-Hochelaga-Maisonneuve, Montréal, Corporation de développement de l'Est, $164 \mathrm{p}$.

Chaline (C.) (dir.), 1994. - Ces ports qui créèrent des villes, Paris, L’Harmattan, 299 p.

Charlier (J.), Malezieux (J.), 1994. - Les stratégies alternatives de redéveloppement portuaire en Europe occidental, Le Havre, Association Internationale Villes et Ports, 96 p.

Cherubini (B.), 1994. - Localisme, fêtes et identités. Une traversée ethno-festive de la Mauricie (Québec), Paris, L'Harmattan, $336 \mathrm{p}$

Debord (G.), 1976. - La société du spectacle, Paris, Champ Libre, 143 p.

Drouin (M.), 2005. - Le combat du patrimoine à Montréal (1973-2003), Québec, Presses de l'Université du Québec, coll. « Patrimoine urbain », 386 p.

Dumazedier (J.), 1962. - Vers une civilisation du loisir?, Paris, Le Seuil, 318 p.

Dufour (C.), 2001. - Mary Travers Bolduc, la turluteuse du peuple, Montréal, Édition XYZ, 187 p.

Fontan (J.-M.), Klein (J.-L.), Tremblay (D.-G.) (dir.), 2005. - Innovation sociale et reconversion économique. Le cas de Montréal, Paris, L'Harmattan, 170 p.

FontAn (J.-M.), YACCARini (C.), 1999. - « Le Technopôle Angus : un exemple communautaire de reconversion industrielle en milieu métropolitain », dans Fontan (J.-M.), Klein (J.-L.), Tremblay (D.-G.) (dir.), Entre la métropolisation et le village global: les scènes territoriales de la reconversion, Sainte-Foy, Presses de l'Université du Québec, coll. «Études d'économie politique », p. 269-288. 
Harter (H.), 2005. - Les pratiques festives au Canada entre espaces privés et publics : un regard historique. Paris, 16 p. Texte de la journée d'études «Fêtes publiques, fêtes privées en Amérique du Nord », organisée conjointement par Centre de Recherche d'histoire Nord Américaine de la Sorbonne et le Centre d'études sur l'Ouest américain et l'Asie-Pacifique anglophone, [http://ameriquedunord.univ-paris1.fr/liberatlantis/fetepubliqueetprivee/fete-publique-et-privee-AN.htm].

Gravari-Barbas (M.), Veschambre (V.) (dir.), 2000. - « Patrimoine et Environnement. Les territoires du conflit », Norois, vol. 47, $\mathrm{n}^{\circ} 185,176$ p.

Klein (J.-L.), Fontan (J.-M.), Tremblay (D.-G.), TARdif (C.), 1998. - « Les quartiers péricentraux : le milieu communautaire dans la reconversion économique », dans Bryant (C.) MANZAGOL (C.) (dir.) 1998. - Montréal 2001, Visages et défis d’une métropole, Montréal, Presses de l’université de Montréal, p. 241-254.

Lefebvre (H.), 1968. - La vie quotidienne dans le monde moderne, Paris, Gallimard, 383 p.

LETTRE UMR ESO, 2005. - Patrimoine et développement durable. Les villes face à la gouvernance patrimoniale. Travaux et documents UMR 6590, Espaces et Sociétés n²3, 140 p.

Manzagol (C.), Bryant (C.) (dir.), 1998. - Montréal 2001, Visages et défis d'une métropole, Montréal, Presses de l'université de Montréal, 356 p.

Micillo Villata (S.), 1995. - Guido Nincheri, maître verrier : Les vitraux des églises Montréalaises, Montréal, Société de Diffusion du Patrimoine Artistique et Culturel des Italo-Canadiens, 79 p.

Muray (P.) 1999. - Après l'histoire, essai, Paris, Les Belles-lettres, 279 p.

Muray (P.), Levy (E.), 2005. - Festivus, festivus, conversation, Paris, Fayard, 485 p.

Mustar (P), Penan (H.), 2003. - Encyclopédie de l'innovation, Paris, Economica, 749 p.

Myles (B.), 2004. - «Six fausses bombes anti-condos Des agitateurs visent des chantiers dans HochelagaMaisonneuve et le Centre-Sud », Le Devoir, 6 janvier, p. Al et 8.

Proulx (J.), 1971. - «Le jeu, le rite, la fête », Critère, n³, janvier cité par l'encyclopédie de l'Agora, [http:// agora.qc.ca/reftext.nsf/Documents/Jeu--Le_jeu_le_rite_la_fete_par_Jean_Proulx].

SÉnÉCAL (G.), SAinT-Laurent (D.) (dir), 2000.- Les espaces dégradés : contraintes et conquêtes, Sainte-Foy, Presses Universitaires du Québec, 272 p.

Simard (M.), Mervier (G.), 2003. - «Négociation sociale, identité communautaire et forme urbaine. La revitalisation du quartier Saint-Roch à Québec », dans Dieudonné (P.), Morisset (L. K.), Simon (J.-F.) (dir.) Réinventer pays et paysages, Brest, Centre de recherche bretonne et celtique/Institut de géoarchitecture de l'Université de Bretagne occidentale, 322 p.

Tremblay (D.-G.), Klein (J.-L.), Fontan (J.-M.), Rousseau (S.), 1998. - « Capital socioterritorial et milieux innovateurs, pistes pour une recherche », dans Côté (S.), ProulX (M.-U.), Espaces en mutation, Rimouski, GRIDEQ, p. 119-133.

VERMEERSCH (L.) 1997. - La reconversion des zones portuaires dans les centres-villes nord-américains; de 1950 à nos jours : vers une redéfinition de la ville moderne, Paris, Université de Paris IV-Sorbonne, 262 p.

Ville de Montréal 1998. - Programme particulier d'urbanisme du Quartier Maisonneuve, Service d'urbanisme de Montréal, 44 p.

—, 2000. - Développement des communautés locales, CDLC HM (Conseil pour le Développement local et communautaire de Hochelaga-Maisonneuve), Montréal, 6 p.

-, 2004. - Plan d'urbanisme de Montréal, Chapitre de Mercier-Hochelaga-Maisonneuve, Direction de l'aménagement urbain et services aux entreprises, Montréal, 66 p.

-, 2005a. - Politique du patrimoine, Montréal, mai 2005, 103 p., [http://ville.montreal.qc.ca/pls/portal/docs/ page/culture_fr/media/documents/politique.pdf].

—, 2005b. - Évaluation du patrimoine urbain de Mercier-Hochelaga-Maisonneuve, Service du Développement culturel, Direction des Activités culturelles, Montréal, 58 p.

Cet article a été reçu le 24 avril 2005 et définitivement accepté le 4 juillet 2006. 